\title{
The Design of Intelligent Password Lock Based on SCM
}

\author{
Bang-qian Ao ${ }^{1, a}, \quad$ Fei-qiao Xiong ${ }^{1, b}$ \\ Engineering institute of Zunyi Normal University, Zunyi City, Guizhou province, \\ China, 563000 \\ aabangqian@163.com , ${ }^{b}$ xfq8925635@163.com
}

Keywords: Password Lock; Function; Key; Live Password.

Abstract: The single chip microcomputer make CPU, RAM, ROM and block PROM, EPROM, EEPROM integrated on a chip of a microprocessor by using a large scale integrated circuit technology. It has multiple I/O port and interrupt system, special function registers, timer, counter, also integrates the driver circuit, pulse width modulation circuit, analog multiplexer and A/D converter, etc. It has the advantages of flexibility, speed, high speed, strong anti-jamming capability, etc. Now, general key password lock password is always constant, unless changing the user or programmer, and some password only can be changed by programmer, it's not very convenient for some users who don't understand the principle. Nowadays, the password lock set no function of self protection, which means that passwords could be easily stolen. The password designed by intelligent password lock based on single chip microcomputer in this article can solve those similar problems.

\section{Introduction}

In this paper, we design a password lock with creativity. It is a kind of live password that can meet demands of users, with dual password function and self protection function. It can well meet the requirements in terms of guard against theft.

First: the design of combination lock:

1. $0 \sim 9$ buttons: Corresponding buttons for $0 \sim 9$;

2. key A: modify password;

3. key B : confirm;

\begin{tabular}{|l|l|l|l|}
\hline 1 & 2 & 3 & A \\
\hline 4 & 5 & 6 & B \\
\hline 7 & 8 & 9 & C \\
\hline$*$ & 0 & $\#$ & D \\
\hline
\end{tabular}

4. key $\mathrm{C}$ : clear;

5. key D : modify the fixed password;

6.key *:modify button lock password;

Fig 1: Matrix keys

7. key \#: quit the modification and clear.

Second: The display design of digital tube: this article uses four digital tube to show the number of those keys, it's easy for the calculation of the latter two number of the live password.
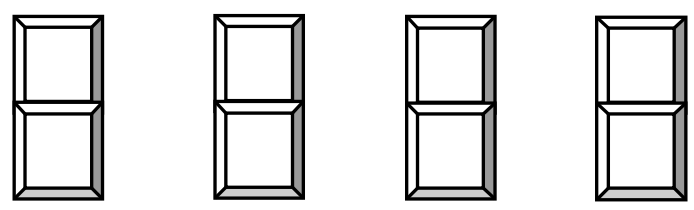

Fig 2: The display of digital tube

Third: The code design:

1. Live password: Six figures. Make up a password with six figures in a certain order, the most 
front four figures is fixed, the last two figures could be changed, each time of opening the combination lock, the last two figures would be changed.

2. Fixed password: six figures. Make up a fixed password with six figures in a certain order.

3. Key lock password: six figures. Make up a fixed password with six figures in a certain order.

4. Special key password: Input digital casually before press the special key, and after press the special key you must input the correct password, then you can continue to input numbers or press the confirm key to open the lock. ( For example: if the special key is 9, and the correct password is 894526, my password could be 4176438989452658964 , then press the confirm key to open the combination lock. The lock can be opened as long as the password satisfies the combination of special key + the correct password.) The advantage of this design is that it can prevent the peep of outsiders and the leak of password. It protects the password in a great extent.

Fourth: the specific design of Special key password:

Press the special key and input the correct password, live password or fixed password, to open the combination lock. The live password is been recommended in general situation. It doesn't matter when strangers saw the enter password and remember it, the latter two number of the password will be changed at the same time when the lock is opened. There is a corresponding display of the latter two numbers. Digital tube display is $\mathrm{I}$, the two live passwords is $\mathrm{I}^{2}$ and $\mathrm{I}^{2}+2$ respectively. For example: if the digital tube display is 3 , then the latter two numbers of the live password is 9 and 1 respectively. We can guarantee the safety of the combination lock in this way. The design of the special key + live password guarantees the safe of the password. Even strangers saw the numbers, they can't know my password correctly. Above function is not the only function of combination lock. If someone input the wrong password in three times, those keys function will be lost. In this case, we should solve the key lock, otherwise combination lock can't open even input the correct password. It has to input the correct key lock password to unlock the key. It can further improve the safety of the combination lock. users can modify the live password, fixed password and key lock password according their own demand. There is only one live password in any time, and so do the fixed password.

Fifth: Password modification design:

1. Press A key: changing the live password .Live password can only be modified the front four fixed number, the latter two can not be modified. Specific operation: Press A key $\rightarrow$ Input the correct fixed password (or live password at this time) $\rightarrow$ Press confirm key B $\rightarrow$ Get corresponding hint, and input a new password(former four number from 0 9 respectively) $\rightarrow$ Press confirm key B $\rightarrow$ Input the new password again (confirm whether the new password in both text). Then press confirm button $\mathrm{B} \rightarrow$ If the new password in both text is consistent, succeed in modification, and back to the original password position $\rightarrow$ If those two new password do not match, modification is not successful. Then you need to press the \# key exit the modifications at this time, back to the original password position. (If you need to continue to modify the password, you can repeat the operation above.).

2. Press the D key: modify the fixed password. Specific operation: Press the D key $\rightarrow$ input the correct fixed password (or live password at this time) $\rightarrow$ press the confirm key B $\rightarrow$ Get corresponding hint, input a new password that has continuous 6 digits $(0 \sim 9)$ at this time $\rightarrow$ press confirm key B $\rightarrow$ input the new password which just entered(confirm that the new password in both text), then press confirm key $\mathrm{B} \rightarrow$ if the new password in both text is consistent, succeed in modification, back to the original password position $\rightarrow$ If those two password do not match, modification is not successful. Then you need to press the \# key exit the modifications at this time, back to the original password position. (If you need to continue to modify the password, you can 
repeat the operation above.).

3. Press the * key: modified fixed password. Specific operation: Press the $*$ key $\rightarrow$ Input correct password $\rightarrow$ Press confirm key B $\rightarrow$ Get corresponding hint, input a new password that has continuous 6 digits $(0 \sim 9)$ at this time $\rightarrow$ press confirm key B $\rightarrow$ input the new password which just entered(confirm that the new password in both text), then press confirm key $\mathrm{B} \rightarrow$ if the new password in both text is consistent, succeed in modification, back to the original password position $\rightarrow$ If those two password do not match, modification is not successful. Then you need to press the \# key exit the modifications at this time, back to the original password position. (If you need to continue to modify the password, you can repeat the operation above.).

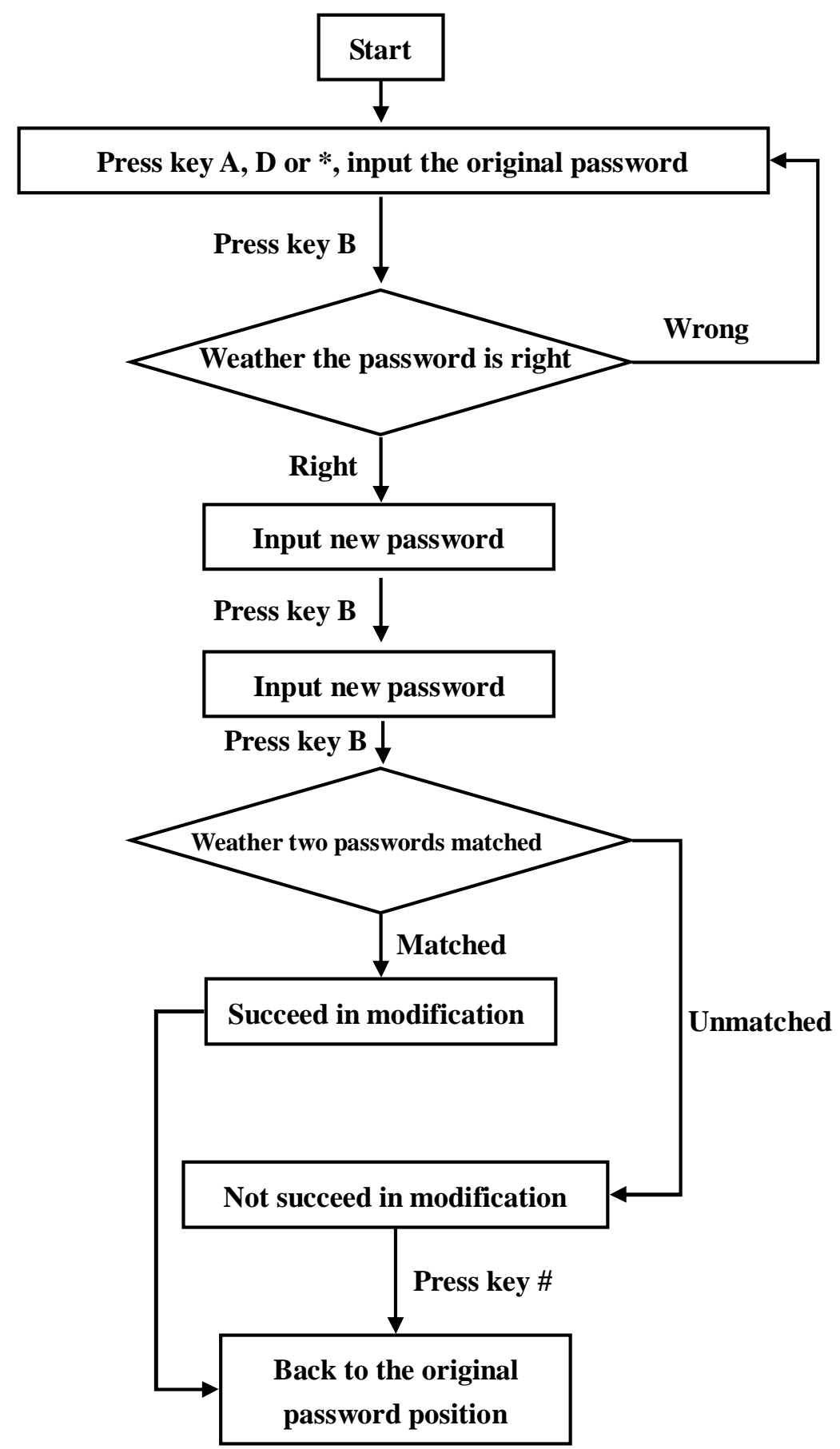

Fig 3: the flow chart of changing the password 
Sixth: the main code of the live password:
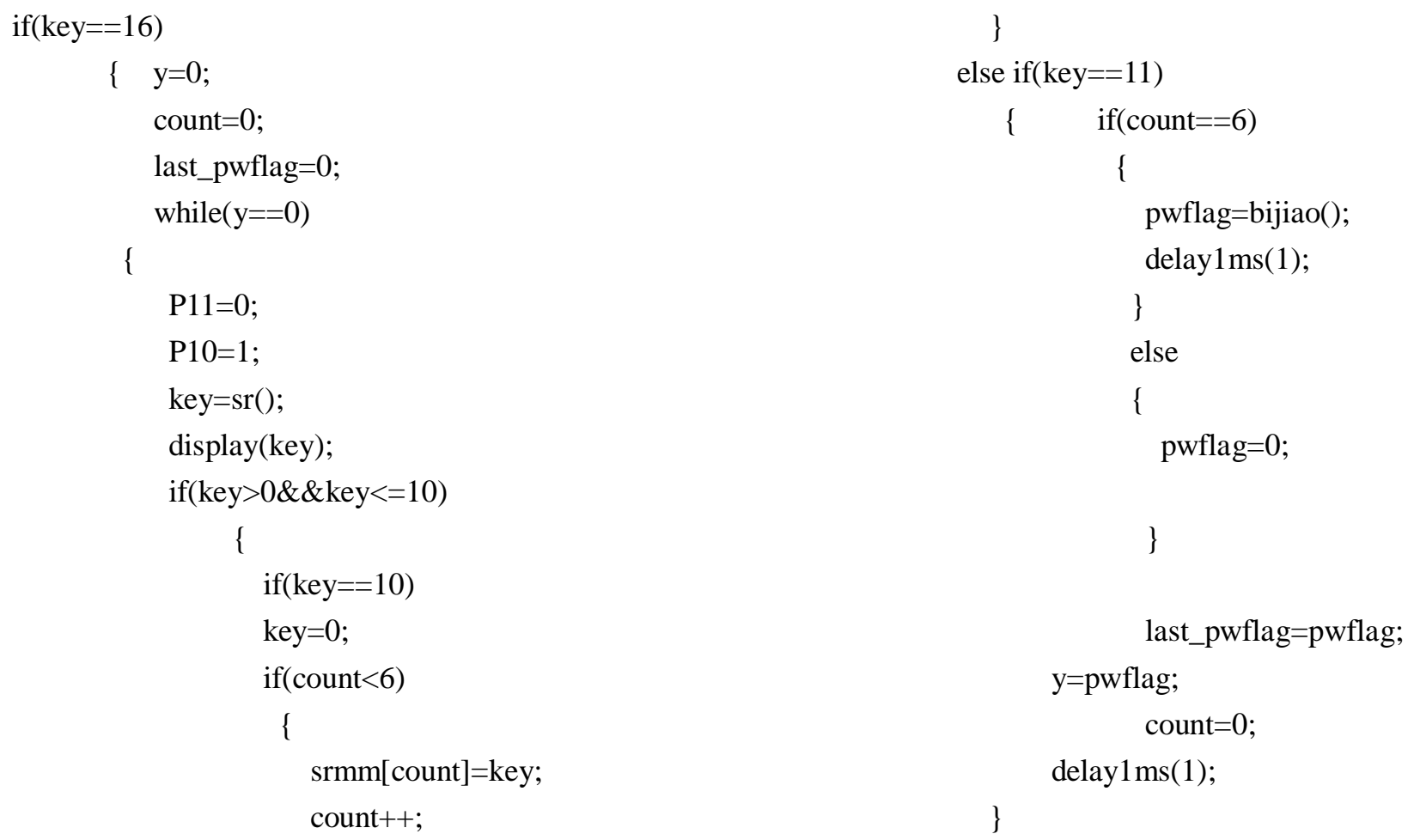

Summary: General key password lock password is always constant, unless changing the user or programmer, and some password only can be changed by programmer, it's not very convenient for some users who don't understand the principle. Nowadays, the password lock set no function of self protection, which means that passwords could be easily stolen. The password designed by intelligent password lock based on single chip microcomputer in this article can solve those similar problems. It is a kind of live password that can meet demands of users, with dual password function and self protection function. It can well meet the requirements in terms of guard against theft

\section{References:}

[1]Pei-ren ZHANG. The Principle and Application of MCS-51 microcontroller based on C language[M], The Tsinghua University Press.

[2]E. Kerr, J. Atwood. The laser illuminated absorptivity spectrophone: a method for measurement of weak absoptivity in gases at laser wavelengths [J].Applied Optics, 1968, (7): 915-921.

[3]L. Kreuzer, N. Kenyon, C. Patel. Air pollution: sensitive detection of ten pollutant gases bycarbon monoxide and carbon dioxide lasers [J] .Science, 1972, (177): 347-349.

[4]Andrew, C.Tam, San Jose. Applications of photoacoustic sensing techniques. Reviews modern Physics, 1986, 862: 381-426. 